\title{
BOGOLIUBOV'S THEORY: A PARADIGM OF QUANTUM PHASE TRANSITIONS
}

\author{
A. M. J. Schakel \\ National Center for Theoretical Sciences, \\ P.O. Box 2-131, Hsinchu, Taiwan 300 \\ Institut für Theoretische Physik, Freie Universität Berlin \\ Arnimallee 14, 14195 Berlin, Germany \\ (Received July 1, 1999)
}

\begin{abstract}
This short essay discusses the application of Bogoliubov's theory of superfluidity in the context of quantum phase transitions.

Key words: Bogoliubov theory, quantum phase transitions, impurities.
\end{abstract}

PACS numbers: 68.35.Rh, 67.40.-w, 67.40.Yv, 72.20.--i

The importance of N. N. Bogoliubov's ground-braking paper On the Theory of Superfluidity [1] in the development of an understanding of superfluidity cannot be underestimated [2]. More than 50 years after the publication of this seminal work, it continues to play a dominant role in contemporary condensed matter physics. It therefore seems appropriate on the occasion of commemorating Bogoliubov's 90th birthday to submit a short essay discussing a modern application of his theory in the context of quantum phase transitions. Some of the material presented here is more extensively discussed in the review [3]. Other recent reviews can be found in Refs. [4-6].

Bogoliubov's theory of superfluidity starts with the Lagrangian

$$
\mathcal{L}=\phi^{*}\left[i \partial_{0}-\epsilon(-i \nabla)+\mu_{0}\right] \phi-\lambda_{0}|\phi|^{4}
$$

where the complex scalar field $\phi(x)$ describes the atoms of mass $m$ constituting the liquid, $i \partial_{0}$ is the total energy operator, while $\epsilon(-i \nabla)=-\nabla^{2} / 2 m$ is the kinetic energy operator, and $\mu_{0}$ the chemical potential. The last term with a positive coupling constant, $\lambda_{0}>0$, represents a weak repulsive contact interaction.

The theory features a global U(1) symmetry, under which the matter field acquires an extra phase factor $\phi(x) \rightarrow \mathrm{e}^{i \alpha} \phi(x)$, with $\alpha$ the transformation parameter. Depending on the ground state, which is determined by the minimum of the potential energy, the symmetry can be realized in two different ways. When $\mu_{0}<0$, the ground state is at $\phi=0$, and the system is in the symmetrical state. As the chemical potential tends to zero, the theory becomes critical, and when $\mu_{0}>0$, the global $\mathrm{U}(1)$ symmetry is spontaneously broken by a nontrivial ground state, given by $|\bar{\phi}|^{2}=\mu_{0} / 2 \lambda_{0}$. This quantity physically denotes the number density $\bar{n}_{0}$ of particles residing in the Bose-Einstein condensate.

The spectrum of the single-particle excitations in this state is given by the celebrated Bogoliubov form [1],

$$
E(\mathbf{k})=\sqrt{\epsilon^{2}(\mathbf{k})+2 \mu_{0} \epsilon(\mathbf{k})}
$$

whose most important signature is that at low momentum it takes the phonon form $E(\mathbf{k}) \sim \sqrt{\mu_{0} / m}|\mathbf{k}|$ predicted by Landau. The spectrum was shown by Beliaev [7] to remain gapless when one-loop quantum corrections are included. And this was subsequently proven to hold to all orders in perturbation theory by Hugenholtz and Pines [8], meaning that the Bogoliubov theory describes a gapless mode. This mode is nothing but the Goldstone mode accompanying the spontaneous symmetry breakdown of the global U(1) symmetry, and is the only degree of freedom present in this state. In other words, the Bogoliubov theory is a phase-only theory. At zero temperature and in the absence of impurities, the phase field is governed by the effective Lagrangian [9]

$$
\begin{aligned}
\mathcal{L}_{\mathrm{eff}} & =-\bar{n}\left[\partial_{0} \varphi+\frac{1}{2 m}(\nabla \varphi)^{2}\right] \\
& +\frac{\bar{n}}{2 m c^{2}}\left[\partial_{0} \varphi+\frac{1}{2 m}(\nabla \varphi)^{2}\right]^{2},
\end{aligned}
$$

where $\bar{n}$ is the average particle number density of the system at rest characterized by a constant phase field $\varphi(x)=$ const, and $c$ is the sound velocity, which to a first approximation equals $c=\sqrt{\mu_{0} / m}$. The phase rigidity in the spatial directions, i.e., the coefficient of $\frac{1}{2}(\nabla \varphi)^{2}$, is seen to be given by $\bar{n} / m$, while that in the temporal direction is given by the compressibility $\kappa$ because

$$
\frac{\bar{n}}{m c^{2}}=\bar{n}^{2} \kappa
$$

Both these rigidities are response functions. Since the chemical potential $\mu$ is represented in the effective theory $(3)$ by $[10]$ 


$$
\mu(x)=-\partial_{0} \varphi(x),
$$

a single differentiation of the effective Lagrangian with respect to $\mu$ yields the particle number density $n(x)=$ $\bar{n}-\left(\bar{n} / m c^{2}\right)\left[\partial_{0} \varphi+(\nabla \varphi)^{2} / 2 m\right]$ of the system slowly varying in space and time

$$
\frac{\partial \mathcal{L}_{\mathrm{eff}}}{\partial \mu(x)}=n(x)
$$

while a second differentiation yields the compressibility

$$
\frac{\partial^{2} \mathcal{L}_{\text {eff }}}{\partial \mu^{2}}=\bar{n}^{2} \kappa
$$

as required. It also follows from Eqs. (5) and (6) that $n$ and $\phi$ are canonically conjugate variables [11]. The form of the effective theory (3), especially the combination $\partial_{0} \varphi+(\nabla \varphi)^{2} / 2 m$ in square brackets is dictated by Galilei invariance [12]. In cases where this symmetry is explicitly broken, as in the presence of impurities and at finite temperature, we expect changes in the relative weights of the coefficients (see below).

Another, for the further development of the theory of superfluidity [2], momentous observation made by Bogoliubov was the so-called depletion of the condensate. He showed that even at the absolute zero of temperature not all the particles reside in the ground state, but [1]

$$
\frac{\bar{n}}{\bar{n}_{0}}-1 \approx \frac{8}{3}\left(\frac{\bar{n} a^{3}}{\pi}\right)^{1 / 2}
$$

where we replaced the coupling constant with the $s$ channel scattering length $a=m \lambda / 2 \pi[13,14]$. (Remember that $\bar{n}_{0}$ denotes the density of particles in the condensate.) Due to the interparticle repulsion, particles are removed from the condensate and put in states of finite momentum. In a strongly interacting system like superfluid ${ }^{4} \mathrm{He}$, the depletion is such that no more than about $8 \%$ of the particles condense in the zero-momentum state [15].

Despite the depletion of the condensate, the phase rigidity in the spatial directions was found in Eq. (3) to be given at the absolute zero of temperature and in the absence of impurities by the total average particle number density $\bar{n} / m$. Since this coefficient denotes the superfluid particle number density $\rho_{\mathrm{s}}$ (divided by $\mathrm{m}^{2}$ ), all the particles - not just those residing in the condensate - participate in the superfluid motion [16]. This changes at finite temperature and also when impurities are included: Galilei invariance is broken then and $\rho_{\mathrm{s}}$ no longer equals $m \bar{n}$. On the other hand, the phase rigidity in the temporal direction as well as the first term in the effective Lagrangian (3) stay the same. This is because relation (5) remains true. In general we thus have as effective theory [17]

$$
\mathcal{L}_{\text {eff }}=-\bar{n} \partial_{0} \varphi-\frac{\rho_{\mathrm{s}}}{2 m^{2}}(\nabla \varphi)^{2}+\frac{1}{2} \bar{n}^{2} \kappa\left(\partial_{0} \varphi\right)^{2}+\cdots
$$

Up to this point we have not specified the external parameter which must be varied to tune the chemical potential to its critical value where the system undergoes a phase transition. In the conventional application of the Bogoliubov theory, the control parameter is the temperature $T$. The critical temperature $T_{c}$ can be determined within the theory by calculating the finite-temperature effective potential and identifying the temperature at which the minimum starts to shift away from the origin. At the one-loop level, one finds [9]:

$$
T_{\mathrm{C}}=\pi\left[\sqrt{2} \zeta\left(\frac{3}{2}\right)\right]^{-2 / 3} \frac{1}{m}\left(\frac{\mu}{\lambda}\right)^{2 / 3}-\frac{2}{3} \frac{\zeta\left(\frac{1}{2}\right)}{\zeta\left(\frac{3}{2}\right)} \mu
$$

where in obtaining this result a high-temperature expansion has been used. This is justified because the leading term is of the order $\lambda^{-2 / 3}$, which is large for weakcoupling. Equation (10) expresses the critical temperature in terms of the chemical potential. From the experimental point of view, however, it is more realistic to have the particle number density as an independent variable. One then finds instead [9]:

$$
\frac{T_{\mathrm{c}}-T_{0}}{T_{0}}=c_{0}\left(\bar{n} a^{3}\right)^{\gamma}
$$

where we again replaced $\lambda$ with the scattering length $a, c_{0}=-\frac{8}{3} \zeta\left(\frac{1}{2}\right) / \zeta\left(\frac{3}{2}\right) \approx 2.82, \gamma=\frac{1}{3}$, and $T_{0}=$ $(2 \pi / m)\left[\bar{n} / \zeta\left(\frac{3}{2}\right)\right]^{2 / 3}$ is the critical temperature of a free Bose gas $(\lambda=0)$. It follows that the critical temperature is increased by the weak repulsive interaction. This is qualitatively different from the strongly interacting ${ }^{4} \mathrm{He}$ system. A free gas with ${ }^{4} \mathrm{He}$ parameters at vapour pressure would have a critical temperature of about $3.1 \mathrm{~K}$, whereas liquid ${ }^{4}$ He becomes superfluid at a lower temperature of $2.2 \mathrm{~K}$. A similar picture emerges from pathintegral Monte Carlo simulations carried out by Grüter, Ceperley, and Laloë [18]. They found that at low densities, corresponding to small $a$, the critical temperature is increased by the repulsive interaction, while at higher densities it is decreased. In the weak-coupling limit, they found numerically the same exponent $\gamma=0.34 \pm 0.03$ as in Eq. (11), while the value of $c_{0}$ was found to be an order of magnitude smaller: $c_{0}=0.34 \pm 0.06$. As argued by these authors, a moderate repulsive interaction suppresses density fluctuations, resulting in a more homogeneous system. This facilitates the formation of large so-called exchange rings necessary to form a BoseEinstein condensate. These exchange rings, as they appear in Feynman's theory of Bose-Einstein condensation [19], consist of bosons which are cyclically permuted in imaginary time (see Ref. [20] for a recent account). At higher densities, the exchange is obstructed because due to the strong repulsive interaction it is more difficult for the particles to move. This leads to a lower critical tem- 
perature.

We now turn to the main subject of this essay, and consider the quantum critical behaviour of the Bogoliubov theory first studied by Uzunov [21]. The critical behaviour of a system close to a quantum phase transition is dominated not by thermal fluctuations as in a classical phase transition at finite temperature, but by quantum fluctuations. In this context, the Bogoliubov theory is considered to be a phenomenological theory similar to the Landau theory of classical phase transitions. The system undergoes a quantum transition at the absolute zero of temperature when the chemical potential approaches the critical values $\mu_{c}$, which is not necessarily zero as in the case of the finite-temperature classical transition. The fine tuning of the chemical potential can be achieved by varying a number of external parameters, such as the charge carrier density, the applied magnetic field, or the impurity strength. For values of the renormalized parameter larger than the critical value $\mu>\mu_{\mathrm{c}}$, the global U(1) symmetry is spontaneously broken and the system is superfluid with a single-particle spectrum given by the gapless Bogoliubov spectrum, implying that the system is compressible. On lowering $\mu$, this state is destroyed and replaced by an insulating state [17].

In the absence of impurities, the insulating state is the so-called Mott-insulator, characterized by the absence of phase rigidity in both spatial and temporal directions, and by an energy gap in the single-particle spectrum. This insulating state, which arises solely due to the repulsive interaction, is consequently incompressible.

On the other hand, in the presence of impurities, the bosons become trapped by the impurities, i.e., Anderson localized. The resulting insulating state is the so-called Bose glass characterized by a single-particle spectrum that is - as in the superfluid state - gapless. This state is therefore also compressible, so that the compressibility remains finite at the transition.

To account for (quenched) impurities, the following term is added to the Bogoliubov theory:

$$
\mathcal{L}_{\Delta}=\psi(\mathbf{x})|\phi(x)|^{2}
$$

with $\psi(\mathbf{x})$ a real random field whose distribution is assumed to be Gaussian [22]

$$
P(\psi)=\exp \left[-\frac{1}{\Delta_{0}} \int \mathrm{d}^{d} x \psi^{2}(\mathbf{x})\right]
$$

and characterized by the impurity strength $\Delta_{0}$. Physically, $\psi$ describes impurities randomly distributed in space. These impurities lead to an additional depletion of the condensate given in $d$ space dimensions by $[23,24]$

$$
\bar{n}_{\Delta}=2^{d / 2-5} \pi^{-d / 2} \Gamma(2-d / 2) m^{d / 2} \lambda^{d / 2-2} \bar{n}_{0}^{d / 2-1} \Delta .
$$

The superfluid and normal mass density $\rho_{\mathrm{s}}$ and $\rho_{\mathrm{n}}$, respectively now become at the absolute zero of tempera- ture [24]

$$
\rho_{\mathrm{S}}=m\left(\bar{n}-\frac{4}{d} \bar{n}_{\Delta}\right), \quad \rho_{\mathrm{n}}=\frac{4}{d} m \bar{n}_{\Delta}
$$

It follows that the normal density is a factor $4 / d$ larger than the mass density $m \bar{n}_{\Delta}$ knocked out of the condensate by the impurities. (For $d=3$ this gives the factor $\frac{4}{3}$ first found in Ref. [25].) As argued by Huang and Meng [25], this implies that part of the zero-momentum states belongs (for $d<4$ ) not to the condensate, but to the normal fluid. Being trapped by the impurities, this fraction of the zero-momentum states are localized. In other words, the phenomenon of Anderson localization can be accounted for in the Bogoliubov theory of superfluidity by including a random field.

The universality class defined by the zero-temperature Bogoliubov theory is not only relevant to describe the critical behaviour of superfluid films (either with or without impurities), but also to describe that of other systems, including Josephson junction arrays and superconducting films. In the so-called composite-boson limit, where Cooper pairs form tightly bound states, the BCS theory directly maps onto the Bogoliubov theory $[26,3]$, which is as we argued a phase-only theory. But even a weakly interacting BCS system was argued to be in the same universality class [27]. The reason is that the amplitude fluctuations of the order parameter are not critical at the transition, not even in the classical superconductor-to-normal transition in $d=3$ [28], only the phase fluctuations are. The phase of the order parameter therefore constitutes the relevant degree of freedom, which is precisely the one described by the Bogoliubov theory. (See, however, Ref. [29], where it is argued that the amplitude fluctuations cannot be neglected, when considering quantum phase transitions in impure superconducting films.) The Bogoliubov theory presumably also forms the basis for the description of the critical behaviour of fractional quantized Hall systems [30].

To investigate the role of quantum fluctuations in the Bogoliubov theory we start with a dimensional analysis. Since, as far as the quantum critical behaviour of this theory is concerned, the mass $m$ is an irrelevant parameter, it can be scaled away by introducing $t^{\prime}=t / m, \mu_{0}^{\prime}=m \mu, \lambda_{0}^{\prime}=\lambda_{0} m$. The engineering dimension of the various variables is then easily determined as:

$$
[\mathbf{x}]=-1, \quad[t]=-2, \quad\left[\mu_{0}\right]=2, \quad\left[\lambda_{0}\right]=2-d, \quad[\phi]=\frac{1}{2} d,
$$

with $d$ the number of space dimensions, and where we dropped the primes again. Note that the time dimension counts double as compared with the space dimensions. This is typical for nonrelativistic theories where the time derivative is accompanied by two space derivatives [see Eq. (1)]. In two space dimensions, the coupling constant $\lambda_{0}$ has a zero engineering dimension, showing that the 
$|\phi|^{4}$-term is a marginal operator, and that $d_{c}=2$ is the upper critical space dimension above which the quantum critical behaviour of the Bogoliubov theory becomes Gaussian. For $d>d_{c}$ quantum fluctuations are irrelevant, while for $d<d_{c}$ these fluctuations become crucial.

Let us next compute the one-loop effective potential

$$
\mathcal{V}_{\mathrm{eff}}=-\frac{\mu_{0}^{2}}{4 \lambda_{0}}+\frac{1}{2} \int \frac{\mathrm{d}^{d} k}{(2 \pi)^{d}} E(\mathbf{k})
$$

with $E(\mathbf{k})$ the gapless Bogoliubov spectrum (2). The integral over the loop momentum yields close to the upper critical dimension $d=2$ :

$$
\mathcal{V}_{\mathrm{eff}}=-\frac{\mu_{0}^{2}}{4 \lambda_{0}}-\frac{1}{4 \pi \epsilon} \frac{m \mu_{0}^{2}}{\kappa^{\epsilon}}+\mathcal{O}\left(\epsilon^{0}\right)
$$

where $\epsilon=2-d$, and $\kappa$ is an arbitrary renormalization group scale parameter, with the dimension of an inverse length. The right-hand side of Eq. (18) is seen to diverge when the upper critical dimension is approached. The theory can be rendered ultraviolet finite by introducing a renormalized coupling constant $\lambda$

$$
\frac{1}{\hat{\lambda}}=\frac{\kappa^{\epsilon}}{\lambda_{0}}+\frac{m}{\pi \epsilon}
$$

where $\hat{\lambda}=\lambda / \kappa^{\epsilon}$. Its definition is such that for arbitrary $d, \hat{\lambda}$ has the same engineering dimension as $\lambda_{0}$ in the upper critical dimension $d=2$. As renormalization prescription we used the modified minimal subtraction. The beta function $\beta(\hat{\lambda})$ follows as [21]

$$
\beta(\hat{\lambda})=\left.\kappa \frac{\partial \hat{\lambda}}{\partial \kappa}\right|_{\lambda_{0}}=-\epsilon \hat{\lambda}+\frac{m}{\pi} \hat{\lambda}^{2} .
$$

In the upper critical dimension, this yields only one fixed point, viz. the infrared-stable (IR) fixed point $\hat{\lambda}^{*}=0$. Below $d=2$, this fixed point is shifted to $\hat{\lambda}^{*}=\epsilon \pi / m$, implying that the system undergoes a 2 nd-order quantum phase transition. Above the upper critical dimension, there is no (nontrivial) renormalization of the coupling constant, which explains why we omitted the subscript 0 on $\mu$ and $\lambda$ in Eq. (10).

Since Eq. (18) could be rendered finite solely by a renormalization of the coupling constant, it follows that the chemical potential is not renormalized to this order. As shown by Uzunov these results remain true to all orders in perturbation theory [21]. The reason for this behaviour is the special analytic structure of the nonrelativistic propagator at criticality, representing only particles propagating forward in time. As a result, the selfenergy (and consequently $\mu$ ) is not renormalized and the full 4 -point vertex function is given by a geometric series, leading to the same beta function (20) found at the one-loop order. For a similar reason, the critical indices characterizing it are Gaussian, despite the nontrivialness of the IR fixed point in $d<2$ [21]. This conclusion was confirmed by numerical simulations in $d=1$ [31].

This changes when impurities are included. A direct application of the renormalization group [32] lead to the conclusion that the IR fixed point becomes unstable. A more careful analysis, using the so-called double epsilon expansion, shows that the fixed point remains stable upon including impurities. The double epsilon expansion was originally introduced in statistical mechanics by Dorogovtsev [33] to treat impurities of finite extend in a classical system. To consistently account for these in perturbation theory, one must assume their dimensionality $\epsilon_{\mathrm{d}}$ to be small, and perform in addition to the usual epsilon expansion, also an expansion in $\epsilon_{\mathrm{d}}$. The impurities described by Eq. (12) are static grains which trace out straight worldlines when time is included. In other words, the impurities are line-like in spacetime, and have also to be treated in a double epsilon expansion, assuming that their dimensionality $\epsilon_{\mathrm{d}}$ is not 1 , but small instead. The quantum critical behaviour of the Bogoliubov theory in $d$ space dimensions with randomly distributed static impurities tracing out "worldlines" of dimensionality $\epsilon_{\mathrm{d}}$ falls in the universality class of a $d$-dimensional classical system with randomly distributed extended impurities of dimensionality $2 \epsilon_{\mathrm{d}}$ - at least to the one-loop order [24]. The factor 2 arises because, as we mentioned before, in the nonrelativistic Bogoliubov theory, time dimensions count double as compared with space dimensions.

Besides having a diverging correlation length $\xi$, 2ndorder quantum phase transitions also have a diverging correlation time $\xi_{t}$, indicating the time period over which the system fluctuates coherently. The way the diverging correlation time scales with the diverging correlation length,

$$
\xi_{t} \sim \xi^{z}
$$

defines the so-called dynamic exponent $z$. The traditional scaling theory of classical 2 nd-order phase transitions is easily extended so as to include the time dimension [22]. Let $\delta \propto K-K_{c}$, with $K$ the external control parameter, denote the distance from the phase transition, so that $\xi \sim|\delta|^{-\nu}$, with $\nu$ the correlation length exponent. At the absolute zero of temperature, a physical observable $O\left(k_{0},|\mathbf{k}|, K\right)$ at finite energy $k_{0}$ and momentum $\mathbf{k}$ can in the critical region be written as

$$
O\left(k_{0},|\mathbf{k}|, K\right)=\xi^{d_{\circ}} \mathcal{O}\left(\xi_{t} k_{0}, \xi|\mathbf{k}|\right), \quad(T=0)
$$

where $d_{O}$ is the scaling dimension of the observable $O$. The right-hand side does depend not explicitly on $K$, but only implicitly through $\xi$ and $\xi_{t}$.

Since a physical system is always at some finite temperature, we have to investigate how the scaling law (22) changes when the temperature becomes nonzero. The easiest way to include temperature in a quantum field theory is to go over to imaginary time $\tau=i t$, with $\tau$ restricted to the interval $0 \leq \tau \leq \beta$, where $\beta=1 / T$ is the inverse temperature. The time dimension thus becomes 
compactified. The critical behaviour of a phase transition at finite temperature is still controlled by the quantum critical point provided $\xi_{t}<\beta$, so that the system does not notice the finite extend of the time dimension. Instead of the zero-temperature scaling (22), we now have the finite-size scaling

$$
O\left(k_{0},|\mathbf{k}|, K, \beta\right)=\beta^{d_{\circ} / z} \mathcal{O}\left(\beta k_{0}, \beta^{1 / z}|\mathbf{k}|, \beta / \xi_{t}\right), \quad(T \neq 0)
$$

The distance to the quantum critical point is measured by the ratio $\beta / \xi_{t} \sim|\delta|^{z \nu} / T$.

Let us apply these general considerations to the effective theory (9) $[34,17]$. The singular part of the free energy density $f_{\text {sing }}$, which scales near the transition as

$$
f_{\text {sing }} \sim \xi^{-(d+z)}
$$

arises from the low-energy, long-wavelength fluctuations of the Goldstone field. The ensemble averages give

$$
\left\langle(\nabla \varphi)^{2}\right\rangle \sim \xi^{-2}, \quad\left\langle\left(\partial_{0} \varphi\right)^{2}\right\rangle \sim \xi_{t}^{-2} \sim \xi^{-2 z}
$$

Combined, these hyperscaling arguments yield the following scaling of the rigidity constants:

$$
\rho_{\mathrm{S}} \sim \xi^{-(d+z-2)}, \quad \bar{n}^{2} \kappa \sim \xi^{-(d-z)} \sim|\delta|^{(d-z) \nu} .
$$

The first conclusion is consistent with the universal jump in the superfluid density predicted by Nelson and Kosterlitz [35] for a Kosterlitz-Thouless phase transition which corresponds to taking $z=0$ and $d=2$.

In an impure system undergoing an Anderson transition, the compressibility $\bar{n}^{2} \kappa$ is nonsingular at the critical point and hence $z=d$ for repulsively interacting bosons in an impure media [34]. Surprisingly, the same conclusion holds for an impure fermionic system [6]. For $d=1$ it follows that space and time appear symmetric as in a relativistic theory.

In a clean system, on the other hand, with a densitydriven Mott transition, i.e., $\delta \propto \mu-\mu_{c}, f_{\text {sing }}$ can also be directly differentiated with respect to the chemical potential to yield for the singular part of the compressibility

$$
\bar{n}^{2} \kappa_{\text {sing }} \sim|\delta|^{(d+z) \nu-2} .
$$

In this case $\bar{n}^{2} \kappa \sim \bar{n}^{2} \kappa_{\text {sing }}$, so that $z \nu=1$ [17] in accord with the Gaussian values $\nu=\frac{1}{2}, z=2$ found by Uzunov [21] for the pure case in $d<2$.

The above hyperscaling arguments have been extended by Fisher, Grinstein, and Girvin [36] to include a $1 /|\mathbf{x}|-$ Coulomb potential. This potential is important for quantum phase transitions in charged systems because the Coulomb repulsion suppresses fluctuations in the charge density and simultaneously enhances those in the canon- ically conjugate variable $\phi$, thereby disordering the ordered state. The quadratic terms of the effective theory in Fourier space after the $1 /|\mathbf{x}|-$ Coulomb potential is included become [36]

$$
\mathcal{L}_{\text {eff }}^{(2)}=\frac{1}{2}\left(\rho_{\mathrm{s}} \mathbf{k}^{2}-\frac{1}{\hat{e}^{2}} k_{0}^{2}|\mathbf{k}|^{d-1}\right)\left|\varphi\left(k_{0}, \mathbf{k}\right)\right|^{2}
$$

where $\hat{e}$ is the renormalized charge. Using similar hyperscaling arguments as before, one finds that this charge scales as

$$
\hat{e}^{2} \sim \xi^{1-z}
$$

Arguing that in the presence of random impurities the charge is nonsingular at the transition, the authors of Ref. [36] concluded that

$$
z=1
$$

This again is an exact result which replaces the value $z=d$ of the neutral system in an impure media.

Most experiments on quantum phase transitions in charged systems measure the conductivity $\sigma$. To describe such type of systems, we minimally couple the Bogoliubov theory to an electromagnetic vector potential $\left(A_{0}, \mathbf{A}\right)$. The conductivity turns out to be related to the superfluid mass density via [27]

$$
\sigma(k)=i\left(\frac{e}{m}\right)^{2} \frac{\rho_{\mathrm{s}}(k)}{k_{0}} .
$$

On account of the scaling relation (26), it then follows that

$$
\sigma \sim \xi^{-(d-2)}
$$

implying that the conductivity and therefore the resistivity is a marginal operator in two space dimensions $[37]$.

The magnetic field $H$ scales with $\xi$ as $H \sim \Phi_{0} / \xi^{2}$, where $\Phi_{0}=2 \pi / e$ is the magnetic flux quantum. This implies that the scaling dimension $d_{\mathbf{A}}$ of $\mathbf{A}$ is unity,

$$
d_{\mathrm{A}}=1
$$

so that $|\mathbf{A}| \sim \xi^{-1}$. From this it in turn follows that the electric field $E=|\mathbf{E}|$ scales as $E \sim \xi_{t}^{-1} \xi^{-1} \sim \xi^{-(z+1)}$, and that the scaling dimension $d_{A_{0}}$ of $A_{0}$ is $z$,

$$
d_{A_{0}}=z
$$

so that $A_{0} \sim \xi_{t}^{-1} \sim \xi^{-z}$.

Let us now be specific and consider quantum phase transitions triggered by changing either the applied magnetic field, i.e., $\delta \propto H-H_{c}$, or the charge carrier density, 


\section{A. M. J. SCHAKEL}

i.e., $\delta \propto n-n_{c}$. For DC $\left(k_{0}=0\right)$ conductivities in the presence of an external electric field $E$ we have on account of the general finite-size scaling form (23) with $k_{0}=|\mathbf{k}|=0$ :

$$
\sigma(K, T, E)=\varsigma\left(|\delta|^{\nu z} / T,|\delta|^{\nu(z+1)} / E\right) .
$$

This shows that conductivity measurements close to a quantum critical point of the kind discussed here should in general collapse onto two branches when plotted as function of the dimensionless combinations $|\delta|^{\nu z} / T$ and $|\delta|^{\nu(z+1)} / E$ : a lower branch bending down for the insulat- ing state and an upper branch tending to infinity for the other state. The best collapse of the data determines the values of $\nu z$ and $\nu(z+1)$. In other words, the temperature and electric-field dependence determine the critical exponents $\nu$ and $z$ independently.

The table below shows experimental data for the critical exponents $z$ and $\nu$ of the superconductor-toinsulator transition in thin films, the Hall-liquid-toinsulator transition in fractional quantized Hall systems, and the conductor-to-insulator transition in silicon MOSFET's at extremely low electron number densities.

\begin{tabular}{c|cc} 
Transition & $z$ & $\nu$ \\
\hline Superconductor-to-Insulator [38,39] & $1.0 \pm 0.1$ & $1.36 \pm 0.05$ \\
Hall-Liquid-to-Insulator [40,41] & $\approx 1.0$ & $\approx 2.3$ \\
Conductor-to-Insulator $[42,43]$ & $0.8 \pm 0.1$ & $1.5 \pm 0.1$ \\
\hline
\end{tabular}

A few remarks seem expedient here. First, the values for the dynamic exponent $z$ found in these systems are in accordance with the prediction $z=1$ recorded in Eq. (30), which was obtained using general hyperscaling arguments for an impure system with a $1 /|\mathbf{x}|-$ Coulomb potential. Second, the values of the critical exponents characterizing the Hall-liquid-to-insulator transition are universal and independent of the filling factor - whether an integer or a fraction. Third, earlier experiments on silicon MOSFET's at lower densities seemed to confirm the general belief, based on the work by Abrahams et al. [44], that such two-dimensional electron systems do not undergo a quantum phase transition. In that paper, where electron-electron interactions were ignored, it was demonstrated that impurities always localize the electrons at the absolute zero of temperature, thus excluding conducting behaviour. Apparently, the situa- tion changes drastically at low electron number densities, where the $1 /|\mathbf{x}|-$ Coulomb interaction becomes important. The values of the critical exponents found for this transition are surprisingly close to those found for the superconductor-to-insulator transition. Since further experiments in an applied magnetic field [45] also revealed a behaviour closely resembling that near the superconductor-to-insulator transition, it is speculated that the conducting state in silicon MOSFET's is in fact superconducting.

\section{ACKNOWLEDGMENTS}

I am grateful to NCTS and B. Rosenstein for the financial support and the hospitality at the Center in Hsinchu, Taiwan, where this work was completed.
[1] N. N. Bogoliubov, J. Phys. USSR 11, 23 (1947).

[2] L. Hoddeson, H. Schubert, S. J. Heims, G. Baym, in Out of the Crystal Maze, edited by L. Hoddeson, E. Braun, J. Teichmann, S. Weart (Oxford University, New York, 1992).

[3] A. M. J. Schakel, in Correlations, Coherence, and Order, edited by D. N. Shopova, D. I. Uzunov (Plenum, New York, 1999), p. 295.

[4] T. Liu, A. M. Goldman, Mod. Phys. Lett. B 8, 277 (1994).

[5] S. L. Sondhi, S. M. Girvin, J. P. Carini, D. Shahar, Rev. Mod. Phys. 69, 315 (1997).

[6] M. Imada, A. Fujimori, Y. Tokura, Rev. Mod. Phys. 70, 1039 (1998).
[7] S. T. Beliaev, Sov. Phys. JETP 7, 289 (1958).

[8] N. M. Hugenholtz, D. Pines, Phys. Rev. 116, 489 (1959).

[9] A. M. J. Schakel, Int. J. Mod. Phys. B 8, 2021 (1994).

[10] P. W. Anderson, Rev. Mod. Phys. 38, 298 (1966).

[11] F. London, Superfluids, Vol. II (Wiley, New York, 1954).

[12] M. Greiter, F. Wilczek, E. Witten, Mod. Phys. Lett. B 3, 903 (1989).

[13] N. M. Hugenholtz, Rep. Prog. Phys. 28, 201 (1965).

[14] A. A. Abrikosov, L. P. Gorkov, I. E. Dzyaloshinski, Methods of Quantum Field Theory in Statistical Physics (Prentence-Hall, Englewood Cliffs, 1963).

[15] O. Penrose, L. Onsager, Phys. Rev. 104, 576 (1956).

[16] P. Nozières, D. Pines, The Theory of Quantum Liquids, Vol. II (Addison-Wesley, New York, 1990). 
[17] M. P. A. Fisher, P. B. Weichman, G. Grinstein, D. S. Fisher, Phys. Rev. B 40, 546 (1989).

[18] P. Grüter, D. Ceperly, F. Laloë, Phys. Rev. Lett. 79, 3549 (1997)

[19] R. P. Feynman, Phys. Rev. 90, 1116 (1953); ibid 91, 1291 (1953).

[20] S. Bund, A. M. J. Schakel, Mod. Phys. Lett. B 13, 349 (1999).

[21] D. I. Uzunov, Phys. Lett. A 87, 11 (1981); Introduction to the Theory of Critical Phenomena (World Scientific, Singapore, 1993).

[22] S. K. Ma, Modern Theory of Critical Phenomena (Benjamin, London, 1976).

[23] S. Giorgini, L. Pitaevskii, S. Stringari, Phys. Rev. B 49, 12938 (1994).

[24] A. M. J. Schakel, Phys. Lett. A, 224, 287 (1997).

[25] K. Huang, H.-F. Meng, Phys. Rev. Lett. 69, 644 (1992).

[26] R. Haussmann, Z. Phys. B 91, 291 (1993).

[27] M. C. Cha, M. P. A. Fisher, S. M. Girvin, M. Wallin, A. P. Young, Phys. Rev. B 44, 6883 (1991); S. M. Girvin, M. Wallin, M. C. Cha, M. P. A. Fisher, A. P. Young, Prog. Theor. Phys. Suppl. 107, 135 (1992).

[28] A. K. Nguyen, A. Sudbø, preprint cond-mat/9907385 (1999).

[29] T. V. Ramakrishnan, Physica Scripta, T27, 24 (1989).

[30] A. M. J. Schakel, Nucl. Phys. B 453, 705 (1995).

[31] G. G. Batrouni, R. T. Scalettar, G. T. Zimanyi, Phys. Rev. Lett. 65, 1765 (1990); G. G. Batrouni, R. T. Scalet- tar, Phys. Rev. B 46, 9051 (1992)

[32] E. R. Korutcheva, D. I. Uzunov, Phys. Lett. A 106, 175 (1984).

[33] S. N. Dorogovtsev, Phys. Lett. A 76, 169 (1980).

[34] D. S. Fisher, M. P. A. Fisher, Phys. Rev. Lett. 61, 1847 (1988).

[35] D. R. Nelson, J. M. Kosterlitz, Phys. Rev. Lett. 39, 1201 (1977).

[36] M. P. A. Fisher, G. Grinstein, S. M. Girvin, Phys. Rev. Lett. 64, 587 (1990).

[37] F. Wegner, Z. Phys. B 25, 327 (1976).

[38] A. F. Hebard, M. A. Palaanen, Phys. Rev. Lett. 65, 927 (1990).

[39] A. Yazdani, A. Kapitulnik, Phys. Rev. Lett. 74, 3037 (1995).

[40] H. P. Wei, D. C. Tsui, M. A. Paalanen, A. M. M. Pruisken, Phys. Rev. Lett. 61, 1294 (1988).

[41] H. P. Wei, L. W. Engel, D. C. Tsui, Phys. Rev. B 50, 14609 (1994)

[42] D. Popovíc, A. B. Fowler, S. Washburn, Phys. Rev. Lett. 79, 1543 (1997).

[43] S. V. Kravchenko, D. Simonian, M. P. Sarachik, W. E. Mason, J. E. Furneaux, Phys. Rev. Lett. 77, 4938 (1996).

[44] E. Abrahams, P. W. Anderson, D. C. Licciardello, T. V. Ramakrishnan, Phys. Rev. Lett. 42, 637 (1979).

[45] D. D. Simonian, S. V. Kravchenko, M. P. Sarachik, V. M. Pudalov, Phys. Rev. Lett. 79, 2304 (1997).

\title{
ТЕОРІЯ БОГОЛЮБОВА: ПАРАДИГМА КВАНТОВИХ ФАЗОВИХ ПЕРЕХОДІВ
}

\author{
А. М. Й. ШШакель \\ Наиіональний иентр теоретичних досліджень, а/с 2-131, Гсінчу, Тайбань 300, \\ Інститут теоретичной фізики, Вілъний університет Берліна, \\ Арнімалле, 14, 14195, Берлін, Німеччина \\ E-mail: schakel@physik.fu-berlin.de

\footnotetext{
У прій короткій статті йдеться про застосування теорії надплинности Боголюбова в контексті теорії квантових фазових переходів.
} 